\title{
Omni-Directional Wideband Antenna Array with Solar Cells
}

\author{
Naveed Ullah ${ }^{1}$ (D) and Ahmet Tekin ${ }^{1}$ (D) \\ ${ }^{I}$ Department of Electrical and Electronics Engineering, Ozyegin University Istanbul, \\ naveed.ullah@ozu.edu.tr,ahmet.tekin@ozyegin.edu.tr
}

\begin{abstract}
Distributed sensor networks are becoming more and more widespread due to their substantial benefits to our daily lives. These sensors, in most cases, are distributed in nature and even may require mobility. This, in turn, implies omnidirectional collector units to collect the data from various sensors at arbitrary directions. This work summarizes the performance outcomes of various integrated wide-band antenna structures and solar power generation topologies to achieve equal gain in all directions. Purpose of the array in this instance is contrary to enhance the received signal levels in all directions. Low-cost solutions ranging from the planar helical printed circuit board (PCB) designs to vertical wire helical antennas were investigated. An optimum design structure for multiple ISM bands $(868 \mathrm{MHz}$ to $915 \mathrm{MHz}$ ) was proposed and characterized considering the cost, size, directional coverage and most significantly RF sensitivity and range of the overall design. The final water-resistant solarpowered wideband transceiver front-end has provided range improvement and omnidirectional radiation pattern. The system consists of a $190 \mathrm{~mm} \times 190 \mathrm{~mm}$ low-cost FR4 substrate and a Wilkinson network to combine the power of 8 helical wire antennas sprinkled along with the solar cells. The self-contained, Sun-powered unit resulted in 4.5-dB sensitivity improvement in measurements.
\end{abstract}

Index Terms - Antenna array, ISM, Solar powered, Wideband

\section{INTRODUCTION}

Wireless Sensor Networks (WSNs) are networks that are formed by a combination of various multipurpose devices, that collect sensory data and transfers them over other network nodes or to a central collector gateway. WSNs were used for military purposes in the beginning. Over time, with the rapid advancements in wireless communication, WSNs became attractive for other applications as well, like, environmental change detection, agriculture, medical, industrial, and the Internet of Things (IoT), most recently[1].

To maximize the performance of a wireless node, a carefully designed and matched antenna frontend is important. The considerable size constraints on wireless nodes induce several trade-offs in the design of such antennas. In this section, some general primers related to such low-cost node antennas are provided together with some general physical limitations on electrically small antennas. In the 
literature, various antenna structures have been proposed for wireless sensor network applications. Dousse, O., and Bettstetter investigated the connectivity of a wireless sensor network through omnidirectional antennas [2]-[3]. Omnidirectional antennas have been analysed by Jun Zhang in further detail [4]. The omnidirectional radiation pattern of a dipole array has been studied by J. Leis group for UHF applications [5]. For low gain variation, a wide beam array is proposed by Yufeng Yu et al.[6]. This antenna has a low gain of about $1.5 \mathrm{~dB}$ and it is used in the particular wireless sensor network. Recent developments in wireless sensor network technology demand highly efficient omnidirectional antennas with horizontal radiation pattern [6].

Moreover, a circular polarized bottom-feed omnidirectional antenna array is proposed in [7]. Half wavelength dipoles and lines loops have chosen and fed from the bottom with the same phase and amplitude. $0.13 \mathrm{GHz}$ measured impedance bandwidth is obtained at $2.45 \mathrm{GHz}$. Also, in [8] an omnidirectional and circular polarized array consisted of four bended dipoles have studied. An array is excited simultaneously by bluns. The array is a good choice however, still due to multilayers and low fractional impedance bandwidth creates complexity. Further low gain antenna study has extended in [9]. Reconfigurable functionality has added in circular polarization with omnidirectional radiation pattern. A very good impedance matching has obtained from $2.1 \mathrm{GHz}$ to $2.5 \mathrm{GHz}$. Similarly, a wide band, compact size and the circular polarized low gain antenna is investigated by Y.M Pan et al., [10]. A circular patch has studied with the modified ground plane for $2.4 \mathrm{GHz}$ centre frequency and $19.8 \%$ bandwidth has achieved. Hence these studies attracted much attention towards wideband and low gain antenna arrays for different applications.

Over recent years several omnidirectional antennas have been investigated. However, in most cases, all the above-mentioned properties can be hardly satisfied for efficient IoT systems. For example, monopoles and vertical dipoles are classical omnidirectional radiation pattern antennas, however, both their bandwidths and gains are limited. Coaxial collinear (COCO) antennas [11]-[12] have gain more than $10 \mathrm{~dB}$, but they are narrow band as well. The gain of $10 \mathrm{~dB}$ can be achieved from a planar slot array with one $\mathrm{dB}$ gain variation but, the bandwidth is only $4.6 \%$ [13]. In reference [14], a back to back planar omnidirectional dipole antenna array is proposed by Wong et al. that are placed symmetrically with respect to the centrally located narrow ground plane. A bandwidth of $5.5 \%$ and a gain of around $5.5 \mathrm{~dB}$ has been attained. Higher gain levels can be achieved by antenna arrays. However, the narrow bandwidth is also a quite serious issue for many applications. Based on these antenna targets, a dual-band dipole planar antenna array [15] has attained $21.9 \%$ and $21.3 \%$ bandwidth and a gain of 6.5 and $6.9 \mathrm{dBi}$ for the 3.5- and 2.6-GHz operating bands, respectively. In another wideband work, GSM850, DCS, and PCS bands are covered by shunt-fed tri-band omnidirectional planar dipole array [16]. Also, a collinear dipole array is designed in [17] at $2.4 \mathrm{GHz}$. However, this design is narrowband and achieved bandwidth is still not enough for some applications. 
Printed antennas have considered being a good candidate for communication due to its low profile structure. Similarly, an ultra-wideband low profile antenna has proposed by R. A. Santos in [18]. This particularly designed antenna covered a very wide range of frequencies from $1.79 \mathrm{GHz}$ to $28 \mathrm{GHz}$. However, the main limitation in this antenna is its low radiation efficiency at low frequencies because of defected ground structure technique.it can be seen that gain is very low as going from high to low frequencies. Similarly another patch antenna array is designed for $2.5 \mathrm{GHz}$ application with the addition of frequency selective surface (FSS) layer. The FSS layer has helped to improve the antenna array performance parameters e.g., bandwidth, gain and radiation efficiency. This in another hand increases the cost and complexity of structure for our proposed idea. Also, the placement of solar cells with patches antenna arrays is a challenging task and will need extra layers for feed network and solar cells that in return will increase the cost of fabrication and will make the structure more complex [19].

In order to overcome many of the above limitations, a printed helical antenna can be used. Aneesh Chowdary Kommalapati, designed a novel printed helical antenna structure for X-band applications [20]. This printed helical antenna can be mathematically modelled by the same formulas that are used for a regular wire helical antenna. In a similar work, a low profile planar helical antenna is designed for circular polarization [21].

16-elements multiple input multiple outputs (MIMO) solar cell combo antenna array system is published by the author for wireless power receiving purposes in the Industrial scientific and medical (ISM) band. The main idea of the mentioned literature work was to make the wireless power system self-power sustained. The antenna array and Coexistence of solar cells were investigated in a limited form factor and designed with less size and cost, yielded an omni-directional horizontal radiation pattern. Each antenna is fed individually and solar cells are kept at good distances from the antennas. As a result, the power of the solar cell is combined with a connected rechargeable lithium battery[22].

In this paper, two types of helical antenna arrays are investigated. One is printed helical antenna arrays with horizontal and vertical ground plane configurations. The second one is wire normal mode helical antenna. The main design requirements are small form factor and omnidirectional radiation pattern. Moreover, in order to isolate the wireless transceiver system from power and data cables, the design had to reuse the ground plane of the antenna array for the placement of solar cells. This structure hence absorbs solar power and recharges the connected lithium battery, resulting in a selfsufficient network element.

\section{ANTENNA SYSTEM DESIGN AND THEORY}

For efficient transmission of information, the antenna is one of the most important devices in a battery-powered wireless network instrumentation hierarchy. While combining multiple sub-systems on a single $\mathrm{PCB}$, the available space for the antennas shrinks. This in return, requires a small size antenna. In this work, multiple wide-band small sizes of omnidirectional helical antenna structures with printed and wire configurations have been investigated.

Brazilian Microwave and Optoelectronics Society-SBMO received 17 May 2020; for review 23 May 2020; accepted 10 Nov 2020 
In three modes (axial mode, normal mode and conical mode) the helical antenna can be operated [23]. Normal mode and printed versions are further studied in this paper in a detailed performance comparison. The helical antenna radiates in the normal direction to the axis when its physical dimensions are less than a wavelength. The radiation pattern of a short-axial-length helix can be determined by assuming that the helix is composed of short dipoles of length $S$ and small loops of diameter $D$. Radiation can be calculated by the equations (1) and (2) shown by Kraus [23]:

$$
E_{\theta}=\frac{j B e^{-j k r}}{r} \frac{S}{\lambda} \sin \theta
$$

The far-field of a short length helix antenna can be determined by assuming the helix consist of small loops and short dipoles. In equation (1) $E_{\theta}$ is the only far-field component of a small loop. While the far-field of a short dipole is represented by $E_{\phi}$ in equation (2). Where both $E_{\theta}$ and $E_{\emptyset}$ components

$$
E_{\varnothing}=\frac{j B e^{-j k r}}{2 r}\left(\frac{\Pi D}{\lambda}\right)^{2} \sin \theta
$$

are out of phase by $90^{\circ}$. Similarly, in both equations $B$ is constant and $r$ is the far-field distance.

The dimensions of a normal-mode helix are small compared with the wavelength, the current distribution is approximately sinusoidal along the helix [24]. The terminal impedance is very sensitive to changes in frequency. Performance of the antenna depends on parameters which include the spacing $(S)$, pitch angle $(\alpha)$ helix heigh $(h)$ and the number of turns $(N)$ which are given in equations (3), (4) and (5) and their values are given in Table.I.

$$
\begin{array}{r}
S<0.05 \lambda \\
h=S \times N<\frac{\lambda}{8}
\end{array}
$$

The pitch angle is defined in (4) as:

$$
\alpha=\tan ^{-1}(S / D)
$$

One of the important parameters is input impedance and can be approximated by equation (5).

$$
Z_{\text {in }}=(140 \times C / \lambda)
$$

In equation (5), $C$ represents circumference.

Hence, it is well established that Normal Mode Helical Antenna (NMHA) input impedance depends very much on wavelength and circumference. By decreasing the helix radius, its input impedance $Z_{\text {in }}$ decreases, and it is inversely proportional to wavelength [20]. Wire helical antenna in this particular system has some limitations; its radiation pattern is deteriorated by the solar cells which are tiled underneath. In order to avoid this drawback, some printed helix topologies were also explored. Printed circuit helix is easy to design and fabricate on PCB and exempted from external support for assembly. Hence, very best advantages can be sited as the volume advantage in the third dimension and no 
additional assembly cost in production. Furthermore, they may take various forms and may be designed in vertical and horizontal with respect to the co-planer ground layer, as it is discussed in section III. Planar helix antennas can be mathematically model by the same formulas provided above that are used for wire helical antennas. The dimension parameters for the design are set according to the criteria $3 / 4<C / \lambda<4 / 3,12^{\circ}<\alpha<14^{\circ}$ and $S<0.02 \lambda[25]-[26]$.

\section{COMPARATIVE STUDY OF ANTENNA ARRAY SOLAR CELL INTEGRATION}

Multiple antennas and solar cell array combo systems are designed, simulated and tested. The details of three types of 8-element design topologies along with solar cells are presented in this section. Solar cells are designed and placed between the mentioned array elements with a $1.5 \mathrm{~mm}$ spacing. Moreover, the cell models consist mainly of two layers; the bottom layer of $2 \mathrm{~mm}$ semiconductor and the top layer of $1 \mathrm{~mm}$ epoxy shield. Total length and width of the single standard solar cell unit is $(65 \times 65 \mathrm{~mm})$. In order to combine the power equally from all eight array elements, a Wilkinson power combiner has been implemented. Trace curvatures and bendings in the layout are adjusted carefully not to impact the RF response.

\section{A. Normal Mode Helical antenna array system}

Initially, a wire antenna array is designed on a FR-4 substrate, utilizing the single element shown in Fig.1. This single element of wire antenna is constructed such that to obtain the required resonance frequency with parameters mentioned in Table. I. Succeeding, the single element is converted into the array of $4 \times 4$ as shown in Fig.2. The total size of the array in the particular design is $190 \mathrm{x} 190 \mathrm{~mm}$ and the substrate height is $1.6 \mathrm{~mm}$. Elements of the array have been placed at $\lambda_{g} / 2$ spacing where $\left(\lambda_{g}\right.$ $=177.12 \mathrm{~mm})$ is the guided wavelength.

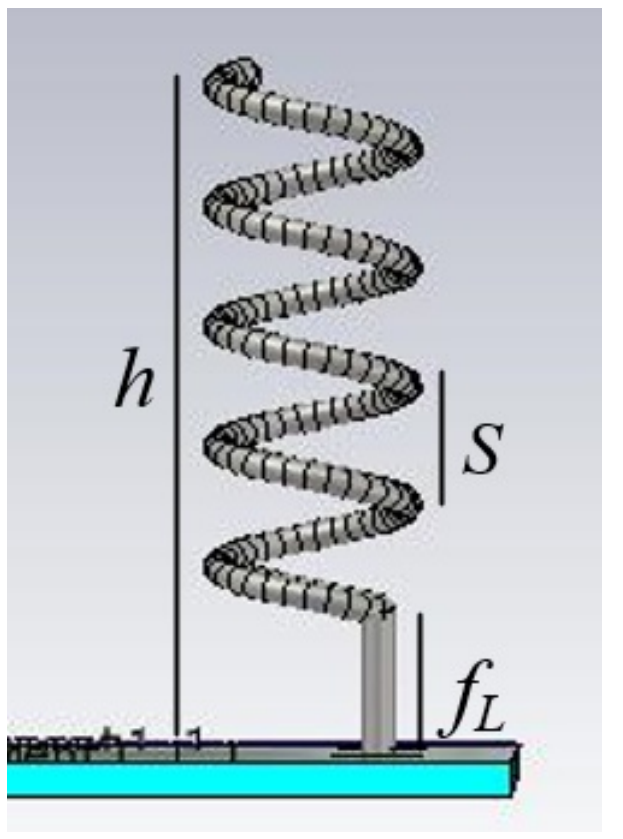

Fig. 1. 3D view of normal mode helical antenna 
TABLE. I. NMHA PARAMETERS

\begin{tabular}{cc}
\hline Parameter & value \\
\hline$N$ & 4.5 \\
$h$ & $26.1 \mathrm{~mm}$ \\
$f_{L}$ & $6.9 \mathrm{~mm}$ \\
$S$ & $5.8 \mathrm{~mm}$ \\
$\alpha$ & $12.7^{\circ}$ \\
Wire radius & $0.65 \mathrm{~mm}$ \\
\hline
\end{tabular}

As mentioned in section II, matching of small helical antenna to a $50 \Omega$ is a challenging task due to its significantly lower input impedance. Using external capacitors and inductors may provide a reasonable matching performance, but, it reduces overall bandwidth [24]. Utilizing a transmission line yielded a slightly better approach. In order to simulate the whole array, firstly, all elements were terminated with 50 s ports and the input impedance of a single element is tuned while the other were actively in place. In this particular configuration, $Z_{\text {in }}$ was found to be in the range of 9.12 to $14.34 \Omega$ real. Following this, the full array performance is evaluated in CST(mw) by placing the antenna feed network and printed helical in xy plane while wire helix is extended in z-axis due to their 3D geometry. The initial NMHA planar antenna system model view with corresponding solar cell fillings is shown in Fig.2.

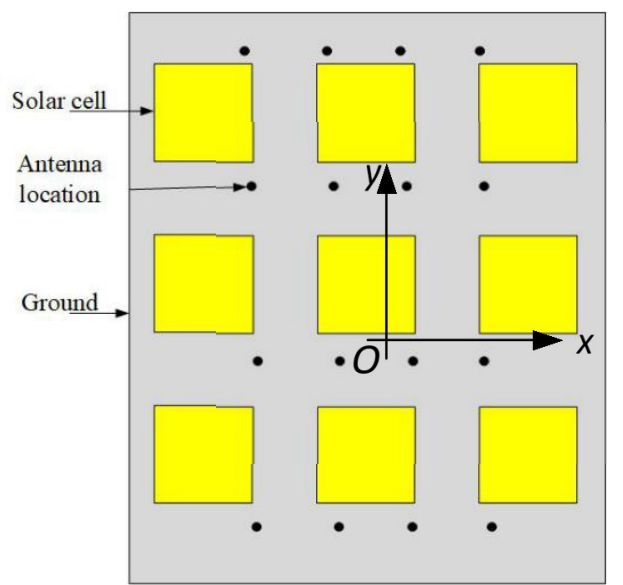

Fig. 2. 16 elements NMHA array filled with distributed solar cells

The main drawback in this 16-element design is its radiation pattern. The radiation pattern of the mentioned antenna array was not as omnidirectional in the horizontal plane as can be seen in Fig. 3 for both elevation and azimuth planes. And the antenna back radiation is comparatively higher for this particular structure. 


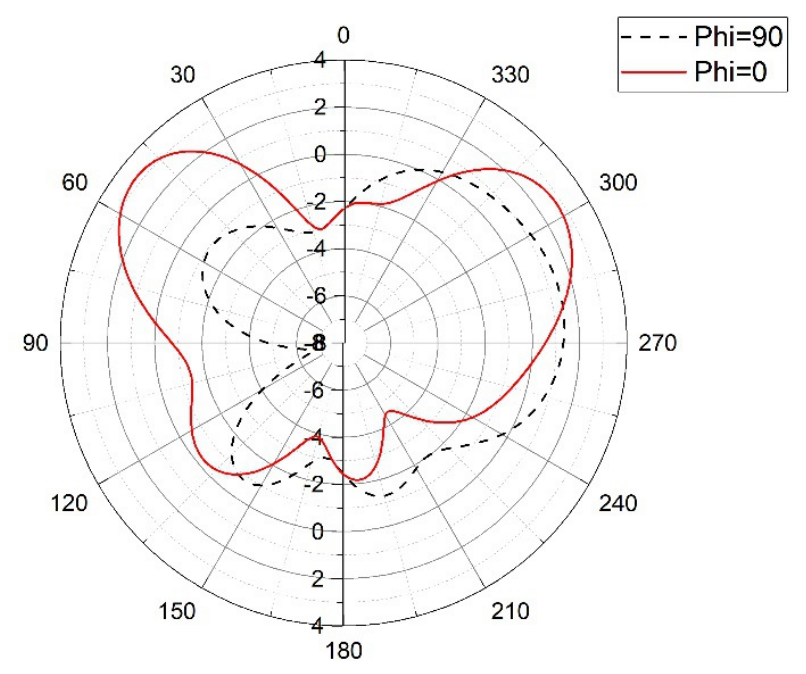

Fig. 3. Radiation pattern plot for wire helical planar antenna array

This design radiates maximum into the vertical plane, which is not as desired in this particular application. The asymmetry is mainly due to the asymmetric distribution of the solar cells only towards one side of each element. Filling the solar cells symmetrically everywhere without any direct ground plane around the elements introduces significant loss, hence a limited asymmetric ground plane was needed. Due to this particular limitation, other topologies were also investigated, horizontal omnidirectional radiation pattern being the main target for range improvement in urban settings.

\section{B. Planar helical antenna array unit design}

Similar to the wire helical antenna array of the previous sub-section, solar cells were sprinkled centrally underneath the vertically stretching antennas between the individual array elements in this planar topology. The loss associated with such a nonperfect ground plane was though significant. The other possibility is to integrate planar helical antenna elements vertical to the corresponding ground plane as shown in Fig. 4. The number of turns in the unit element design is 17, spacing is $1.54 \mathrm{~mm}$, single-arm length and width are 16.2 and $1 \mathrm{~mm}$, respectively. The total helix size of the single unit is $60 \mathrm{~mm}$. The best arrangement even, in this case, requires a significant separation between elements and solar cells. Hence, the solar cells are placed on the same plane corresponding to an area at the centre of the ground plane away from the individual antenna array elements. The Wilkinson combiner tree is constructed on the other side of the low-cost double layer PCB, sharing the same ground plane. This vertically aligned antenna array is shown in Fig.5. 


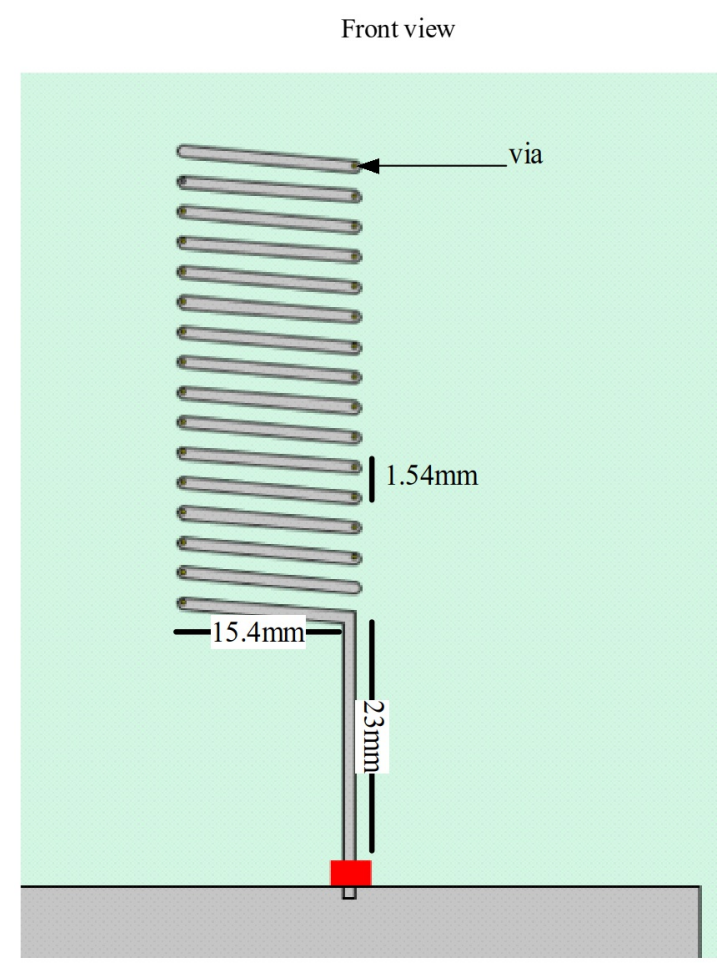

Back view

Fig. 4. Planar helix

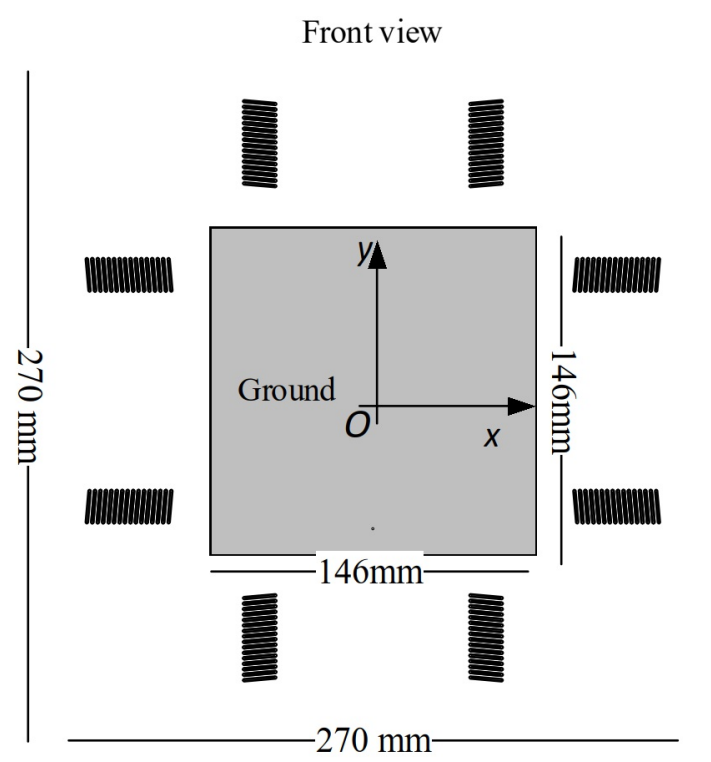

Back view

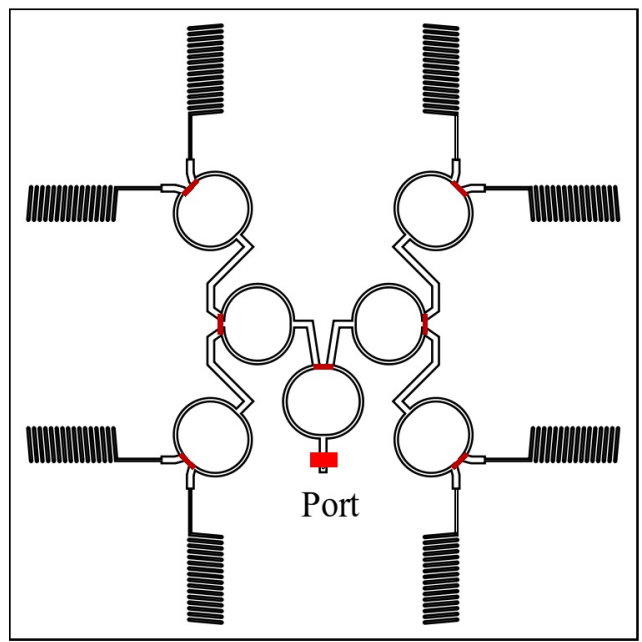

Fig. 5. Vertically distributed planar helix array

Feed length and hence the distance to the ground plane played an important role in antenna resonance frequency. The simulation result for input return loss $\left(\mathrm{S}_{11}\right)$ is shown in Fig. 6. It can be depicted from the plot that the antenna provided good matching at $868 \mathrm{MHz}$. The radiation pattern plot is also shown in Fig.7 for this 8-element array. The first drawback of this particular system is that the bandwidth is not wide enough to cover all the high-ISM bands in the range 868-MHz to $929-\mathrm{MHz}$. The other and even more undesirable characteristic is the radiation null in the horizontal plane, and 
more back radiated power for both azimuth and elevation planes which is undesired and opposes the target application's main specification.

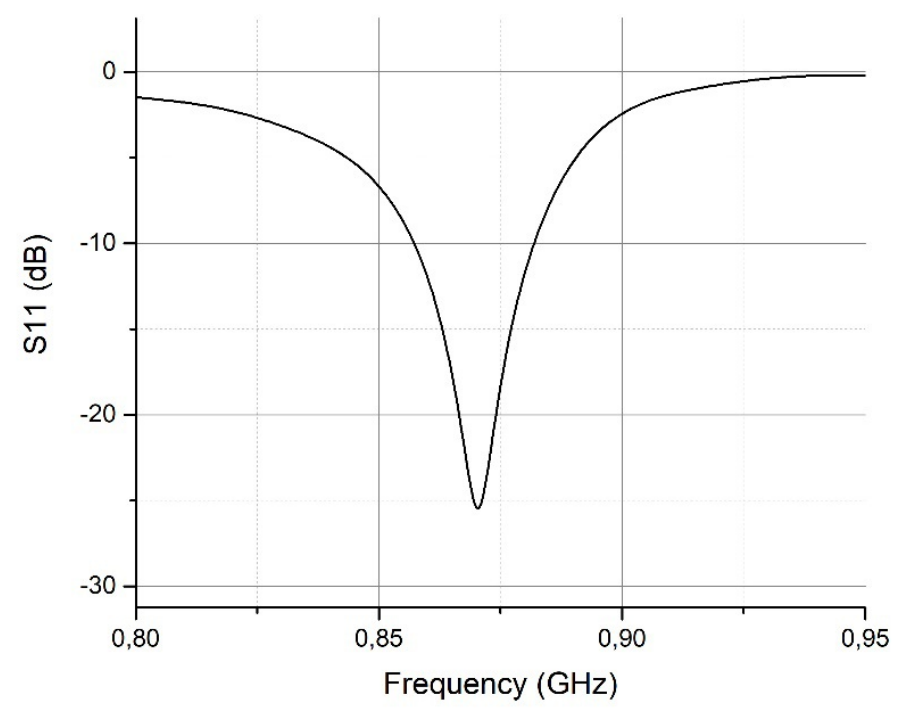

Fig. 6. Simulated return loss plot of the vertically located printed helical array

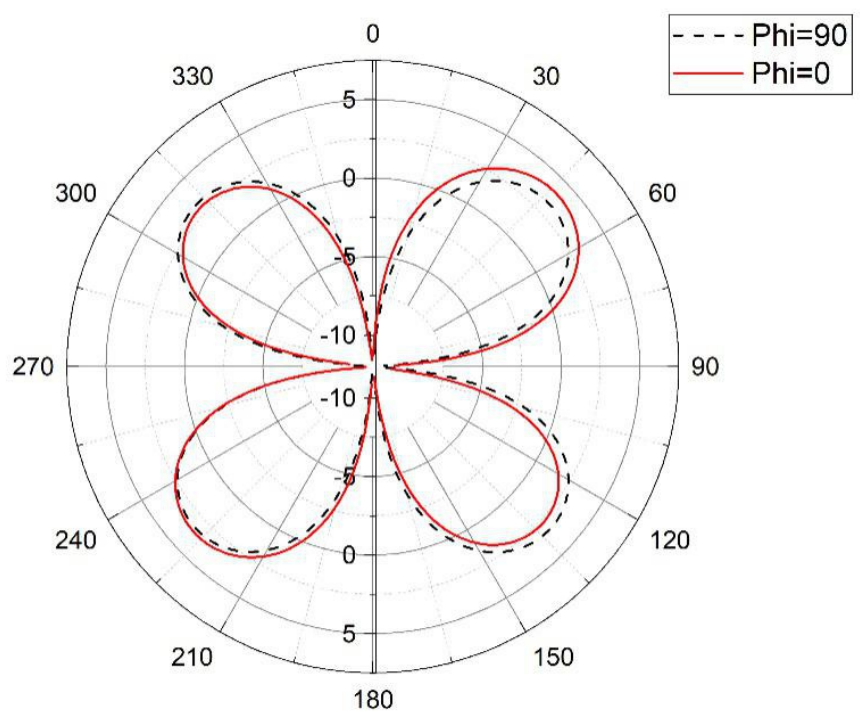

Fig. 7. Radiation pattern for the vertical planar helical array

In order to alleviate this particular back-radiation problem and improve the pattern in the horizontal planes, horizontally referenced printed helical antenna performance was also investigated. This horizontally aligned printed helical antenna individual element details, as well as full 8-element array configuration along with the Wilkinson tree, are shown in Fig. 8 and Fig.9 respectively. 


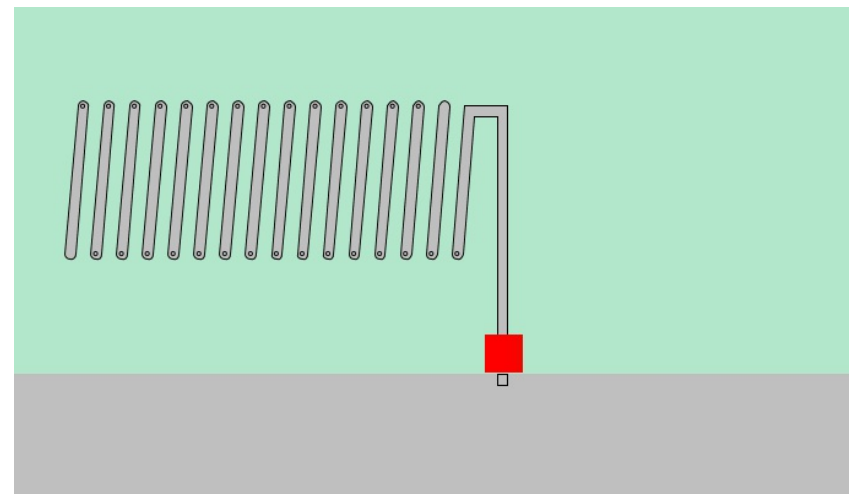

Fig. 8. Horizontally aligned planar helix unit element

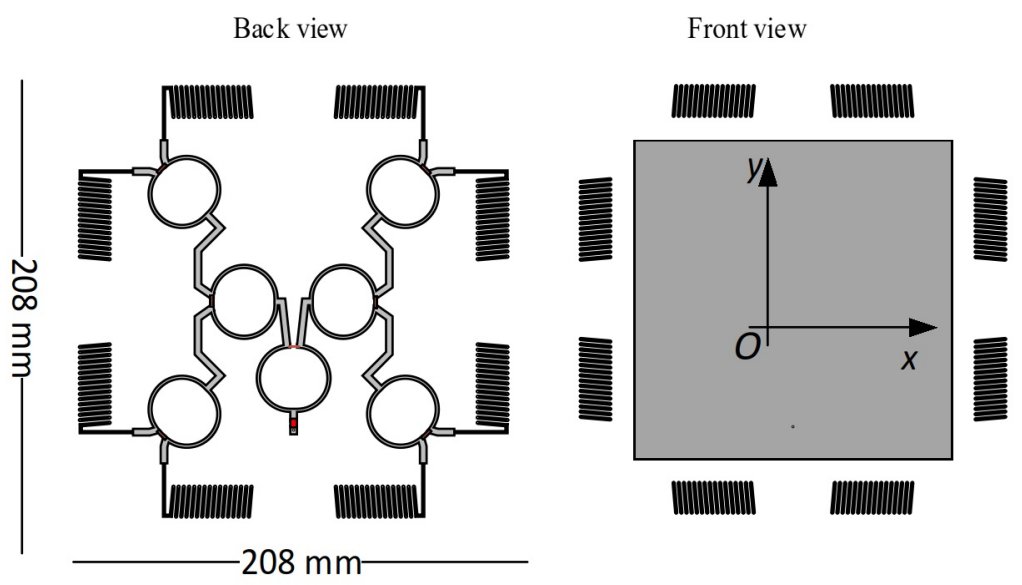

Fig. 9. Planar helical array of horizontal alignment with respect to the ground plane

This array system geometry although has significantly improved the horizontal radiation pattern as shown in Fig. 10, most of its energy is still directed towards the top and bottom in the vertical axis.

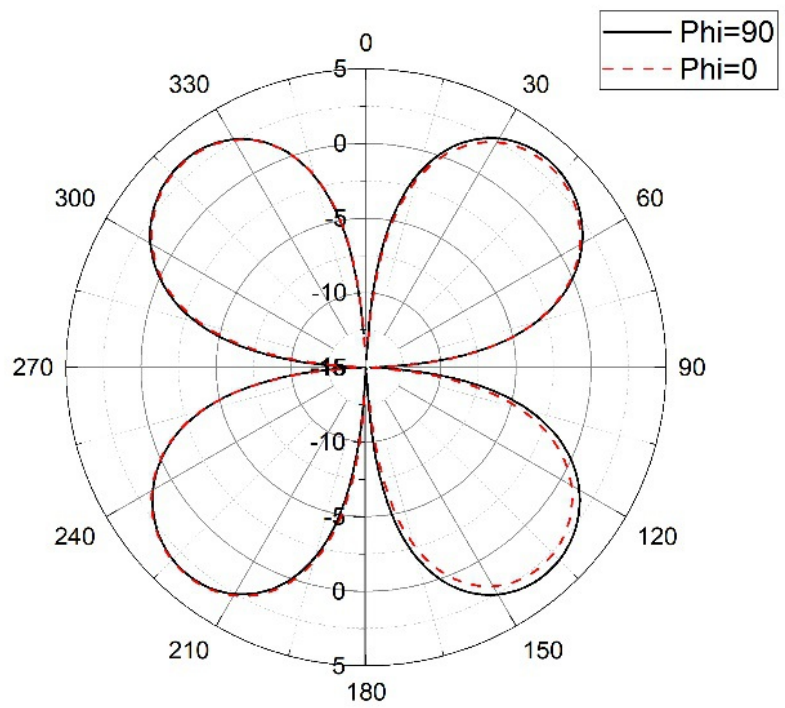

Fig. 10. Radiation pattern of the horizontally referenced PCB helical antenna array 
C. NMHA circular array with central solar cells

Due to the drawbacks related to the previously discussed three topologies, a final configuration of 8-element design with centrally placed solar cells was reached as the most appropriate structure for an omnidirectional horizontal pattern wideband IoT antenna array. This particular system layout is shown in Fig. 11. It employs eight vertical helical antennas lined up in a circular track around the periphery with a maximum distance away from the central ground plane where solar cells are all pulled towards the centre to minimize the associated radiation losses. On the top layer where the antennas are, there is the ground plane and the bottom trace level of the PCB hosts the Wilkinson power combining tree.

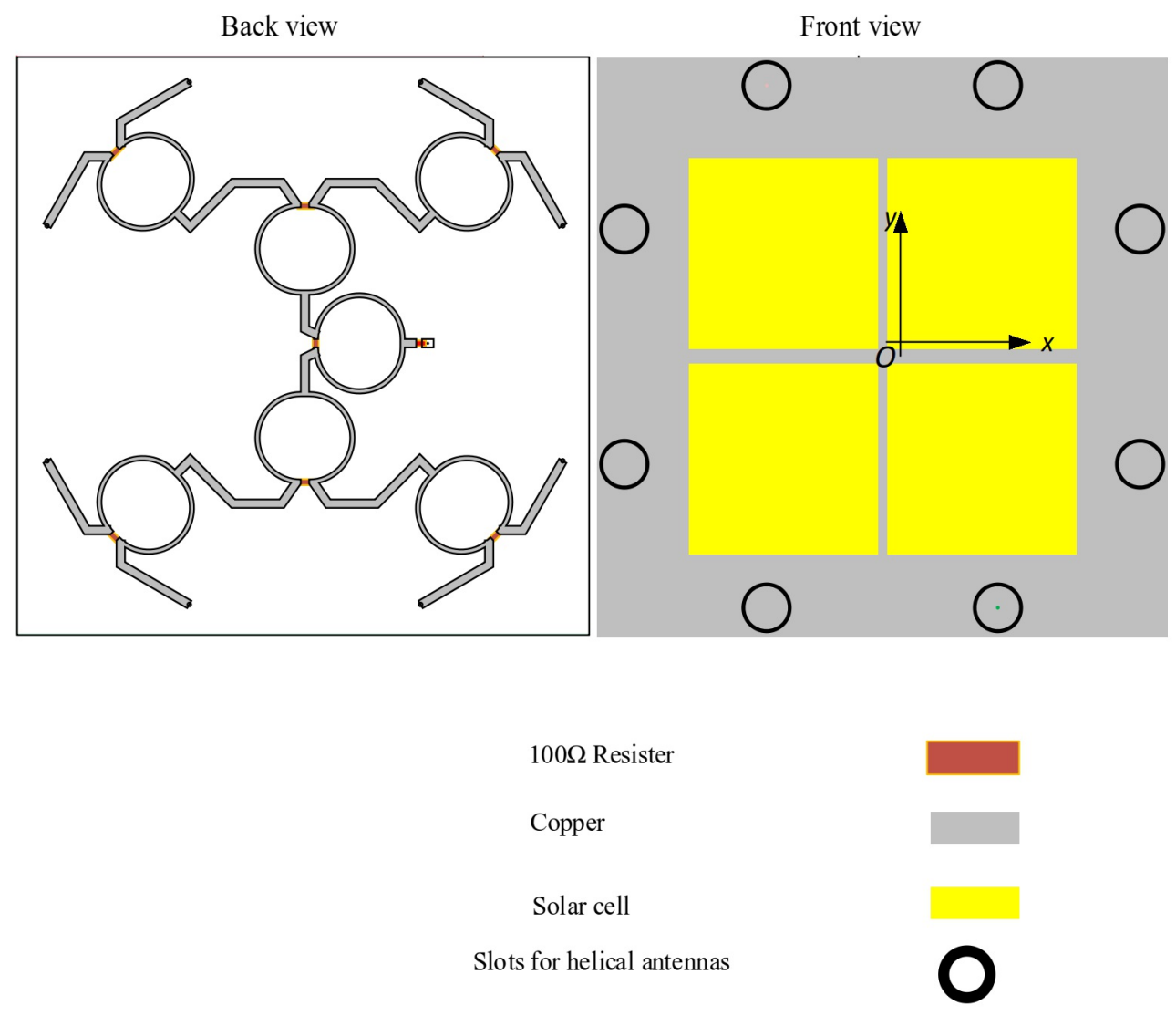

Fig. 11. Circular distributed normal mode helical antenna array geometry

In order to prove the target performance metrics of the proposed NMHA antenna array, a prototype is fabricated and tested as shown in Fig. 12. The dimensions of the unit wire helix prototype are the same as the one described in section III. The return loss of the NMHA array is measured with the help of the EB720 Network Analyzer as shown in the measurement setup presented in Fig. 12(a) and fabricated prototype in Fig. 12(b). 


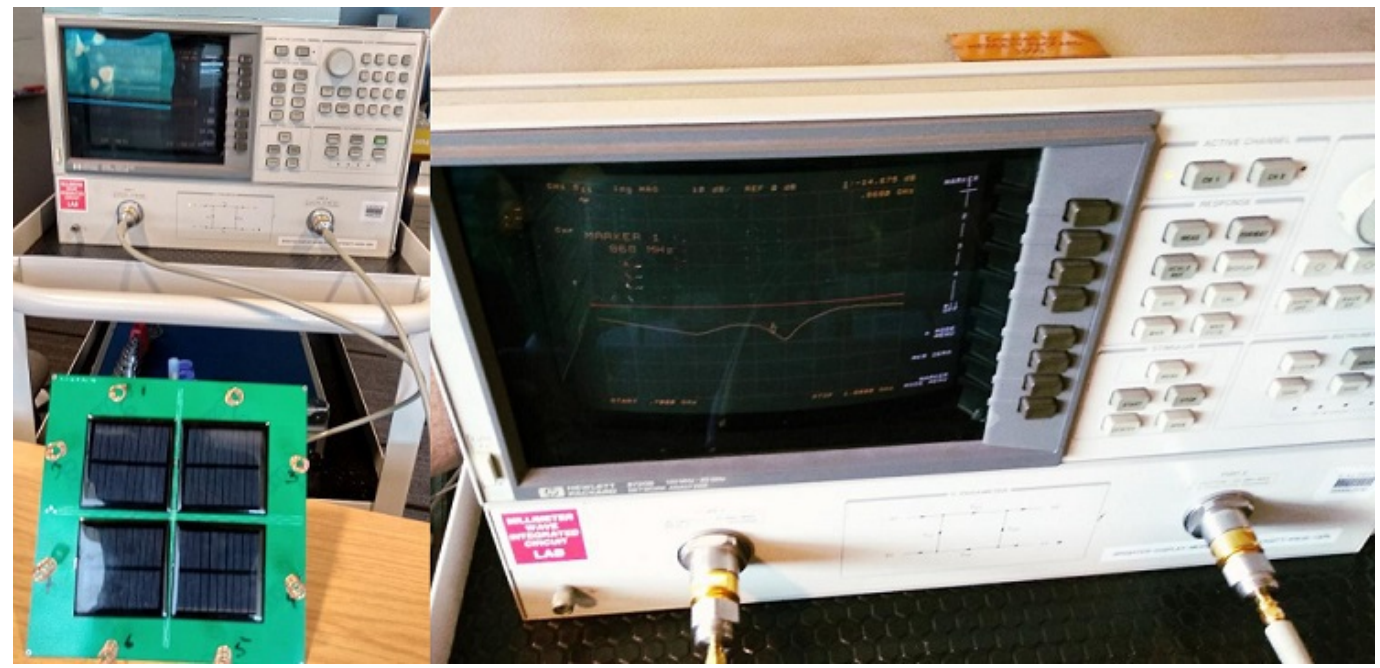

(a)

Front view

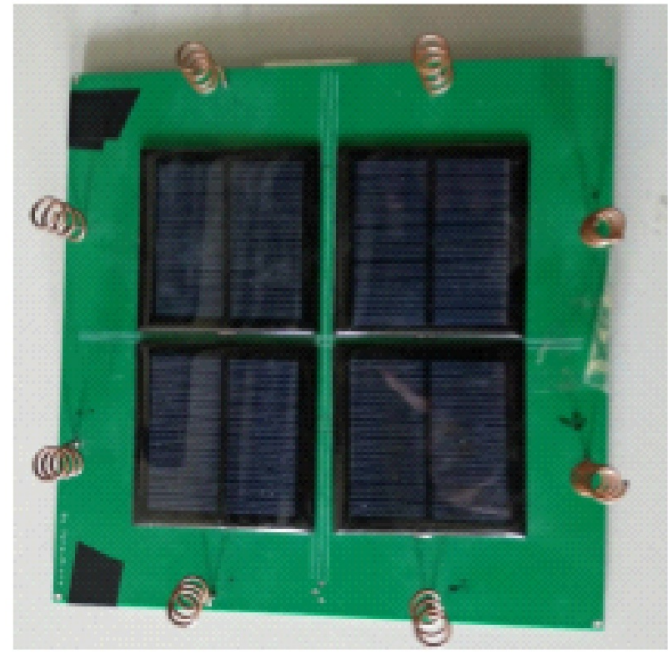

Back view

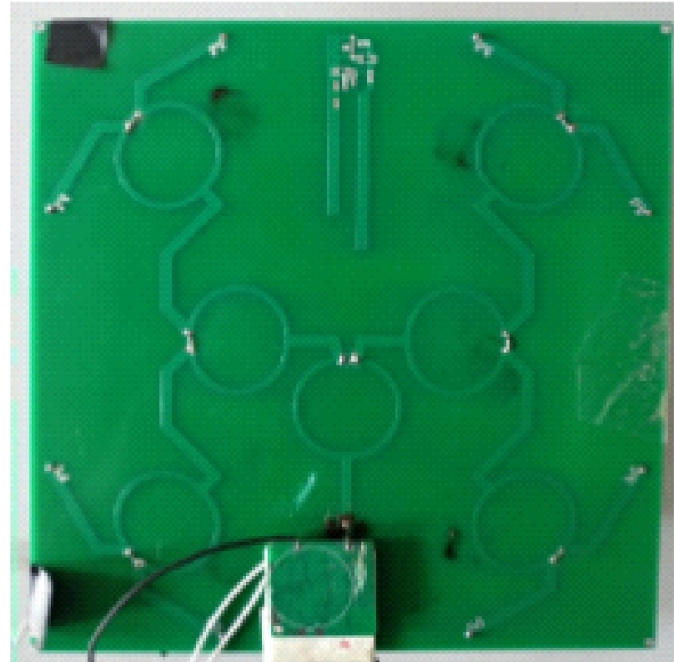

(b)

Fig. 12. (a) Measurement setup view; (b) Front and back view of the fabricated Prototype

The measurement results of the reflection coefficient $\left(S_{11}\right)$ are presented and compared with the corresponding simulated results. The measured reflection coefficient $(S 11)$ is plotted in Fig. 13. As can be seen from Figure the input impedance of the proposed helical antenna array is stable across a wide frequency range with a matching impedance of $50 \Omega$, and hence it provides a relatively wide impedance bandwidth. The simulated impedance bandwidth $\left(S_{11}<-10 \mathrm{~dB}\right)$ ranges from $790 \mathrm{MHz}$ to $940 \mathrm{MHz}(17.2 \%)$ while the default untuned measured impedance bandwidth covers a range from $710 \mathrm{MHz}$ to $915 \mathrm{MHz}$ (23.61\%). Difference between simulated and measured reflection coefficient is observed and there are several reasons behind this mismatching. For example, in simulation, a discrete 50 -ohm port is used to excite the antenna without inserting connector. However, in measured a real 50- ohm connector is integrated. Similarly, coaxial cable is used while connecting antenna to vector network analyzer that also creates a difference between measured and simulation results due to coaxial cable behaviours. Comparative results are given in Table. II. As compared to the results Brazilian Microwave and Optoelectronics Society-SBMO received 17 May 2020; for review 23 May 2020 ; accepted 10 Nov 2020 
obtained in [13], [14], [16] and [17] there is an improvement of $18.1 \%, 17.8 \%, 15.59 \%$ and $11.6 \%$ in achieved BW by proposed circular helical antenna array topology.

TABLE. II. COMPARATIVE RESULTS FOR OMNI DIRECTIONAL ANTENNA ARRAYS

\begin{tabular}{cccc}
\hline Reference & Frequency & BW (\%) & Gain (dBi) \\
\hline$[13]$ & $5.8 \mathrm{GHz}$ & 5.5 & 5.5 \\
{$[14]$} & $2.4 \mathrm{GHz}$ & 5.8 & 6.1 \\
{$[16]$} & $824 \sim 894 \mathrm{MHz}$ & 8.01 & $3.8 \sim 5.5$ \\
{$[17]$} & $2.4 \mathrm{GHz}$ & 12 & 7.75 \\
{$[22]$} & $900 \mathrm{MHz}$ & 2.2 & 2.2 \\
Proposed design & $868 \mathrm{MHz}$ & 23.61 & 2.6 \\
\hline
\end{tabular}

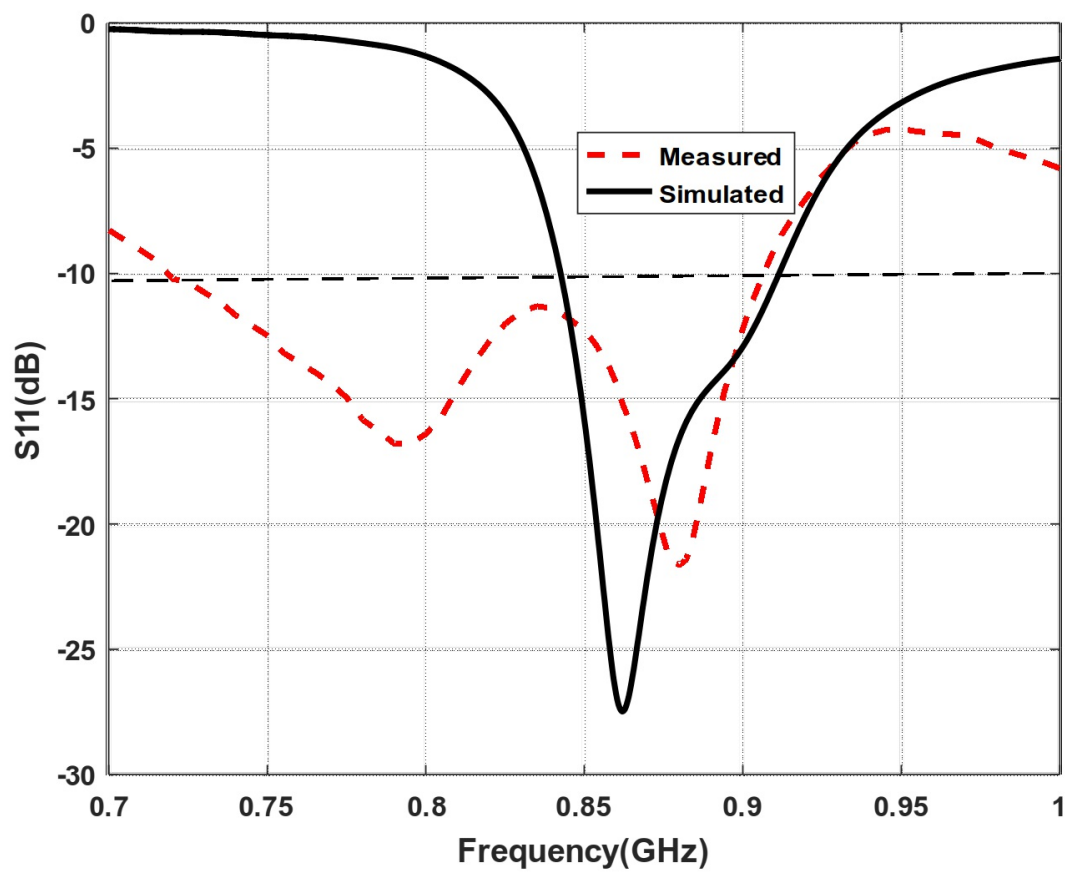

Fig. 13. Simulated and measure s-parameter plot 


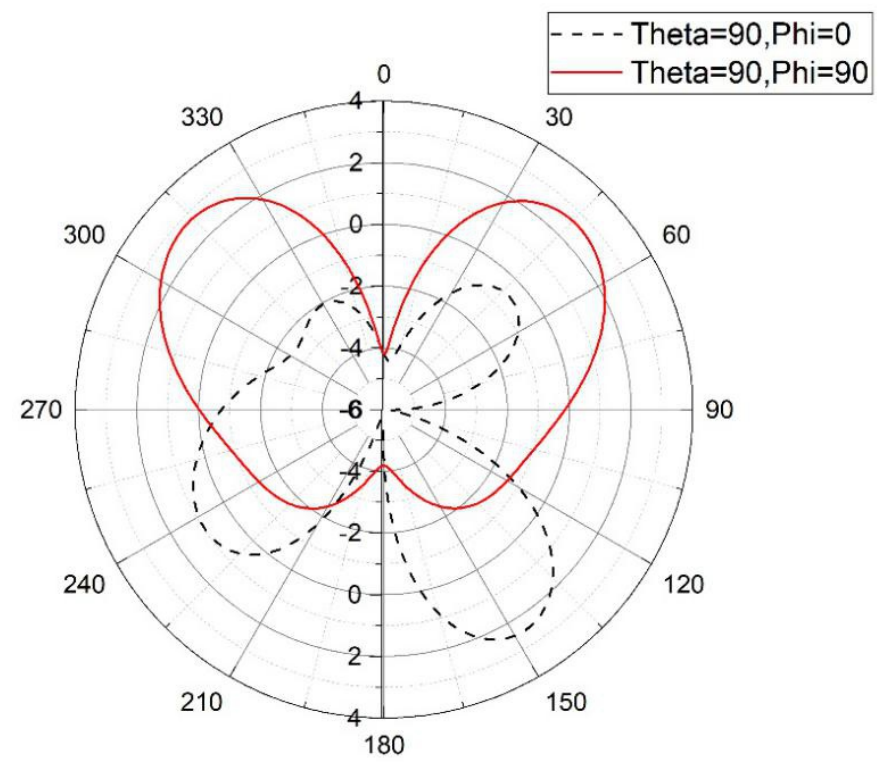

Fig.14. Radiation pattern for the proposed circular array

Fig. 14 shows that the circular antenna array radiation is highest in the horizontal plane with respect to other combo topologies that required special horizontal placement for solar charging. The same figure shows a minimal back-radiation level with respect to the other topologies, which in return makes it the most suitable structure for an IoT outdoor concentrator unit.

Furthermore, the receiver signal to noise ratio (SNR) of the single helical antenna and the proposed antenna array with the distributed network has been measured by spectrum analyzer and compared as shown in Fig.15. It was observed that the SNR value of the received signal in the case of the array configuration had $4.5 \mathrm{dBm}$ better performance than a single helical antenna. This had improved communication range significantly in the final system range tests.

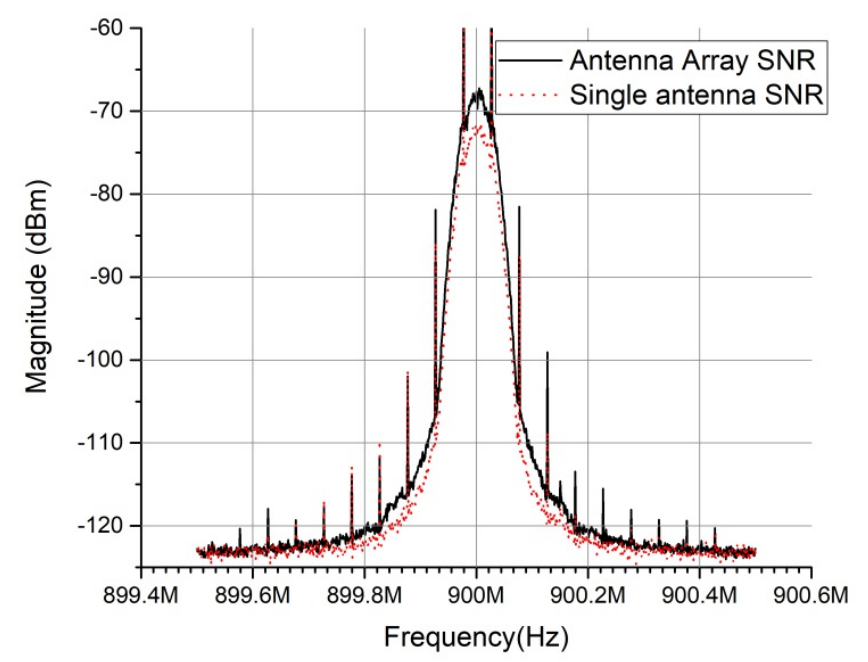

Fig.15. SNR Comparison between single elements and array of the helical wire antenna 


\section{CONCLUSION}

Three different types of helical antenna geometrical and structural topologies are analyzed to reach an omnidirectional antenna array at $868 \mathrm{MHz}$, in combination with solar cells. Various optimizations have been carried out for all of the corresponding design cases. It is concluded that maximum distance circular array geometry was the best option with the most optimal radiation pattern while co-existing with the solar cells. Overall, VSWR of the antenna array and distribution network is less than 1.5 around the centre of the target frequency range. In addition to the immediate benefit of this particular design in providing better SNR, even more, pronounced benefit lays in the fact that it can be a waterresistant and solar-charged outside unit. Furthermore, it can provide even more sensitivity advantages considering the height, wall losses and other loss factors associated with the current indoor gateway systems.

\section{REFERENCES}

[1] V. Butenko, A. Nazarenko, N. S. and Viliam Sarian, and A. Lutokhin, "Applications of Wireless Sensor Networks in Next Generation Networks," Ser. T.2000 Next Gener. Networks, no. February, pp. 1-94, 2014.

[2] O. Dousse, S. Member, and I. IEEE, François Baccelli, and Patrick Thiran, Member, "Impact of Interferences on Connectivity in Ad Hoc Networks," ACM Int. Conf. Proceeding Ser., vol. Part F1306, no. 2, pp. 40-44, 2017, doi: $10.1145 / 3129186.3129194$.

[3] L. Jianxin, L. Yuanzhen, L. Tonghong, Z. Xiaomin, and Z. Lei, "On the connectivity of one-dimensional vehicular ad hoc networks," China Commun., vol. 7, no. 2, pp. 65-70, 2010.

[4] J. Zhang and X. Jia, "Capacity analysis of wireless mesh networks with omni or directional antennas," Proc. - IEEE INFOCOM, pp. 2881-2885, 2009, doi: 10.1109/INFCOM.2009.5062251.

[5] J. Lei, G. Fu, L. Yang, and D. M. Fu, "An omnidirectional printed dipole array antenna with shaped radiation pattern in the elevation plane," J. Electromagn. Waves Appl., vol. 20, no. 14, pp. 1955-1966, 2006, doi: $10.1163 / 156939306779322639$.

[6] Y. Yu, J. Xiong, and R. Wang, “A Wideband Omnidirectional Antenna Array With Low Gain Variation,” vol. 15, pp. 386-389, 2016.

[7] X. Li, L. Yang, J. W. Zhao, Y. Liu, and F. Z. Sun, “A bottom-feed omni-directional circularly polarized antenna array," Int. J. Microw. Wirel. Technol., vol. 11, no. 10, pp. 1075-1080, 2019, doi: 10.1017/S1759078719000825.

[8] Y. Yu, J. Xiong, and H. Li, “Compact omni-directional circularly polarised antenna utilising bended dipoles and integrated baluns," IET Microwaves, Antennas Propag., vol. 11, no. 10, pp. 1409-1414, 2017, doi: 10.1049/ietmap.2016.0947.

[9] Y. M. Cai, S. Gao, Y. Yin, W. Li, and Q. Luo, "Compact-Size Low-Profile Wideband Circularly Polarized Omnidirectional Patch Antenna With Reconfigurable Polarizations," IEEE Trans. Antennas Propag., vol. 64, no. 5, pp. 2016-2021, 2016, doi: 10.1109/TAP.2016.2535502.

[10] Y. M. Pan, S. Y. Zheng, and B. J. Hu, "Wideband and low-profile omnidirectional circularly polarized patch antenna,” IEEE Trans. Antennas Propag., vol. 62, no. 8, pp. 4347-4351, 2014, doi: 10.1109/TAP.2014.2323412.

[11] T. J. Judasz and B. B. Balsley, "Improved theoretical and experimental models for the coaxial colinear antenna," IEEE Trans. Antennas Propag, 2018.

[12] J. F. Kiang, "Analysis of linear coaxial antennas," IEEE Trans. Antennas Propag., vol. 46, no. 5, pp. 636-642, 1998, doi: $10.1109 / 8.668905$.

[13] X. Chen, K. Huang, and X. B. Xu, "A novel planar slot array antenna with omnidirectional pattern," IEEE Trans. Antennas Propag., vol. 59, no. 12, pp. 4853-4857, 2011, doi: 10.1109/TAP.2011.2165481.

[14] K. L. Wong, F. R. Hsiao, and T. W. Chiou, "Omnidirectional planar dipole array antenna," IEEE Trans. Antennas Propag., vol. 52, no. 2, pp. 624-628, 2004, doi: 10.1109/TAP.2004.823897.

[15] J.-H. Lu and Y.-H. Liu, "PLANAR DUAL-BAND DIPOLE ARRAY FOR LONG-TERM EVOLUTION/ WORLDWIDE INTEROPERABILITY FOR MICROWAVE ACCESS POINTS,” Microw. Opt. Technol. Lett., vol. 55, no. 11, pp. 2562-2568, 2013, doi: 10.1002/mop.

[16] K. Wei, Z. Zhang, W. Chen, Z. Feng, and M. F. Iskander, "A triband shunt-fed omnidirectional planar dipole array," IEEE Antennas Wirel. Propag. Lett., vol. 9, pp. 850-853, 2010, doi: 10.1109/LAWP.2010.2069077.

[17] K. G. Thomas and N. Lenin, "COLLINEAR PRINTED ARRAY ANTENNA," Microw. Opt. Technol. Lett., vol. 55, no. 11, pp. 2562-2568, 2013, doi: 10.1002/mop.

[18] R. A. Santos and S. Arismar Cerqueira, "A low-profile and ultra-wideband printed antenna with a $176 \%$ bandwidth,” J. Microwaves, Optoelectron. Electromagn. Appl., vol. 16, no. 1, pp. 59-69, 2017, doi: 10.1590/2179$10742017 \mathrm{v} 16 \mathrm{i} 1621$.

[19] V. A. Almeida Filho and A. L. P. S. Campos, "Performance optimization of microstrip antenna array using

Brazilian Microwave and Optoelectronics Society-SBMO received 17 May 2020; for review 23 May 2020 ; accepted 10 Nov 2020 
Journal of Microwaves, Optoelectronics and Electromagnetic Applications, Vol. 20, No. 1, Month 2021

DOI: http://dx.doi.org/10.1590/2179-10742021v20i1885

frequency selective surfaces," J. Microwaves, Optoelectron. Electromagn. Appl., vol. 13, no. 1, pp. 31-46, 2014, doi: $10.1590 / \mathrm{S} 2179-10742014000100003$.

[20] A. C. Kommalapati, C. Zhao, and S. Aditya, "A printed planar helix antenna," 2015 9th Eur. Conf. Antennas Propagation, EuCAP 2015, pp. 19-22, 2015.

[21] Z. Chen and Z. Shen, "Planar helical antenna of circular polarization," IEEE Trans. Antennas Propag., vol. 63, no. 10, pp. 4315-4323, 2015, doi: 10.1109/TAP.2015.2463746.

[22] N. Ullah and A. Tekin, "16-Elements Helical Antenna System Integration with a Solar Cell Powered IoT Collector," Proc. - CAMA 2019 IEEE Int. Conf. Antenna Meas. Appl., pp. 69-72, 2019, doi: 10.1109/CAMA47423.2019.8959623.

[23] A. T. Adams and J. K. Lee, "Helical Antennas," Encycl. RF Microw. Eng., pp. 1-26, 2005, doi: 10.1002/0471654507.eme159.

[24] L. Huang, J. Xiong, and Y. Yu, "An electrically small normal-mode helical antenna with capacitive coupling feed," 8th Eur. Conf. Antennas Propagation, EuCAP 2014, no. EuCAP, pp. 2915-2917, 2014, doi: 10.1109/EuCAP.2014.6902436.

[25] C. A. Balanis, Antenna theory: analysis and design. John wiley \& sons, 2016.

[26] C. Su, H. Ke, and T. Hubing, "A simplified model for normal mode helical antennas," 2010. 